\title{
Variability in the expression of polycomb proteins in different normal and tumoral tissues. A pilot study using tissue microarrays
}

\author{
Margarita Sánchez-Beato ${ }^{1}$, Esther Sánchez ${ }^{2}$, Joaquín González-Carreró ${ }^{\text {, }}$ Manuel Morente ${ }^{4}$, \\ Ana Díez ${ }^{5}$, Lydia Sánchez-Verde ${ }^{5}$, María Carmen Martín ${ }^{6}$, Juan C Cigudosa ${ }^{6}$, Miguel Vidal ${ }^{7}$ \\ and Miguel A Piris ${ }^{1}$ \\ ${ }^{1}$ Lymphoma Group, Molecular Pathology Programme, Centro Nacional de Investigaciones Oncológicas (CNIO), \\ Madrid, Spain; ${ }^{2}$ Department of Pathology, Hospital 'Nuestra Señora del Prado', Talavera de la Reina-Toledo, \\ Spain; ' ${ }^{3}$ Department of Pathology, 'Complejo Hospitalario Universitario de Vigo' (CHUVI)', Vigo-Pontevedra, \\ Spain; ${ }^{4}$ Tumour Bank, Molecular Pathology Programme, Centro Nacional de Investigaciones Oncológicas \\ (CNIO), Madrid, Spain; ${ }^{5}$ Immunohistochemistry and Histology Unit, Biotechnology Programme, Centro \\ Nacional de Investigaciones Oncológicas (CNIO), Madrid, Spain; ${ }^{6}$ Cytogenetics Unit, Biotechnology \\ Programme, Centro Nacional de Investigaciones Oncológicas (CNIO), Madrid, Spain and ${ }^{7}$ Department of \\ Developmental and Cell Biology, Centro de Investigaciones Biológicas, (CSIC), Madrid, Spain
}

In spite of the known function of polycomb group (PcG) genes in stem cell self-renewal, control of cellular proliferation and differentiation, its role in cancer pathogenesis is still poorly understood. We studied the expression by immunohistochemistry of several PcG-maintenance complex proteins (RING1, RNF2, BMI1, MEL18, HPH1 and RYBP) in nontumoral (154 samples) and tumoral (550 samples) human tissues using Tissue Microarrays. For selected genes (BMI1 and RING1) FISH analysis has been also carried out. PcG proteins had a tissue- and cell-type-specific expression pattern. Some of them were highly selectively expressed, such as HPH1, which was detected in germ cells in testis, pituitary and parathyroid glands and Langerhans islets, and RYBP, which was found in placenta, umbilical cord and thyroid gland. By contrast, RING1 was ubiquitously expressed in every normal tissue analyzed. Changes in expression associated with tumoral transformation have been found for BMI1 and RNF2, which exhibited increased expression in a large series of tumors, including gastrointestinal tumors, pituitary and parathyroid adenomas, and lymphomas, compared with their expression in normal-cell counterparts. The high level of expression of BMl1 protein observed in mantle-cell lymphomas and pituitary adenomas is associated in some cases with amplification of BMI1 locus. These findings imply that upregulation of BMI1 may constitute a malignancy marker in different types of cancer, mainly in lymphoid and endocrine tumors. RING1 was lost in a group of renal-cell carcinomas and testicular germ-cell tumors. Lastly, RYBP is anomalously expressed in Hodgkin's lymphomas and oligodendrogliomas, among others tumors. A significant finding of the study is the identification of unique PcG profiles for some tumors, such as testicular germ-cell tumors, which have high levels of HPH1 expression and loss of RING1 and/ or BMI1; pituitary adenomas, which expressed every PcG protein analyzed; and clear-cell renal-cell carcinoma, which was the only tumor other than testicular germ-cell tumors that did not express RING1.

Modern Pathology (2006) 19, 684-694. doi:10.1038/modpathol.3800577; published online 10 March 2006

Keywords: polycomb; cancer; tissue-microarray; immunohistochemistry; FISH

Many different processes regulate cellular differentiation, proliferation arrest and, eventually, cell death. Neoplastic transformation is the result

Correspondence: Dr M Sánchez-Beato, PhD, Molecular Pathology Program, Centro Nacional de Investigaciones Oncológicas (CNIO), C/Melchor Fernández Almagro No. 3, E-28029 Madrid, Spain. E-mail: msbeato@cnio.es

Received 14 September 2005; revised and accepted 19 January 2006; published online 10 March 2006 of deregulation of any of these processes that lead to abnormal proliferation of the cells. Chromatin structure and modifications and, therefore, chromatin regulatory factors, are involved in the control of cell growth and, consequently, in tumorigenesis. Among these factors, the polycomb group (PcG) constitutes an important set of proteins that regulate gene activity at the chromatin level. ${ }^{1-3}$ In Drosophila, in which they were first described, two groups of proteins were found to be responsible for 
the maintenance of homeotic gene activity in the appropriate segments during fly development: PcG itself, and the trithorax group (trxG).

PcG proteins form DNA-binding protein complexes, and although the composition of these is variable, two biochemical and major functional PcG complexes have been described in humans.

- The Polycomb Repressive Complex 1 (PRC1), or PcG-maintenance (PcGm), which organizes the chromatin into a repressive structure. ${ }^{4-8}$ MEL18, BMI1, RING1, RNF2, HPH1, HPH2, HPC1, HPC2 and HPC3 are members of this complex. Recent studies identify a PRC1-like complex as the H2A ubiquitin ligase, linking $\mathrm{H} 2 \mathrm{~A}$ ubiquitination to PcG silencing. ${ }^{9}$

- The PRC2 and PRC2-like complexes, which have histone methyl transferase activity, also known as PcG-initiating (PcGi), contain the proteins ENX/ EZH, EED and SUZ12, among others. ${ }^{4,10}$

Although the two complexes seem to have opposite roles with respect to cellular survival and proliferation, they are thought to function concertedly to maintain the repressed status of the gene. First, PRC2 methylates K27 and/or K9 of histone H3 and PRC1 complex recognizes this marker ${ }^{11}$ binding to chromatin.

Many different PcG complexes have been characterized that also contain non-PcG proteins such as transcriptions factors, ${ }^{12,13}$ histone methyltransferases ${ }^{11}$ and histone deacetylases. ${ }^{14}$ The composition of these complexes has been postulated to depend on cellular type and context. ${ }^{10,15,16}$

RYBP was identified by its direct interaction with RING1, RNF2 and YY1 proteins. ${ }^{8}$ RYBP is thought to belong to the PcG family, based on data from genetic analysis that implies a PcG function for this protein (MV, unpublished observations).

Defects of several of these proteins in transgenic mice have been shown to produce skeletal and hematopoietic defects and other specific alterations. For example, mice transgenic for Bmi1, Mel18 and M33 genes show hematopoietic anomalies such as severe spleen and thymus hypoplasia, ${ }^{17-19}$ and Rnf2 deficiency results in an early lethal phenotype. ${ }^{20}$

Studies of PcG in human tissues show that this system plays important roles in several biological functions, such as regulation of cell differentiation, ${ }^{16,18,21,22}$ hematopoietic and neural stem cell self-renewal ${ }^{23-25}$ and control of cellular proliferation. $3,17,18,21,26-29$

The role of PcG in human cancer is mostly unknown, but some evidence indicates the involvement of this system in tumorigenesis. Recent studies have demonstrated the importance of certain proteins in several types of human tumor. This is the case for EZH2 (with histone methyl transferase activity), which is involved in progression in prostate cancer and in neoplastic transformation of breast epithelial cells, ${ }^{30-32}$ and murine Bmi1, which collaborates with c-Myc in transforming lymphoid cells. ${ }^{33-35}$ Human BMI1 has been found to be deregulated in mantle cell lymphomas, ${ }^{36,37}$ in Hodgkin's and in diffuse large B-cell lymphomas. ${ }^{38-42} \mathrm{~A}$ high level of expression of BMI1 has also been described in medulloblastomas, ${ }^{43}$ colorectal carcinoma, ${ }^{44}$ and a misregulation of BMI1 was shown to be inversely correlated with INK4/ARF in NSCLC. ${ }^{45}$ However, the existing studies have largely focused on lymphomas and leukemias, ${ }^{26,37,38,46,47}$ and no exhaustive analysis has hitherto been made of tumors of different origin.

Our aim was to analyze PcGm protein expression in a broad spectrum of tumoral samples, and to compare it with the expression in their corresponding normal counterparts, with the ultimate aim of investigating the role of this PcGm complex in human tumorigenesis.

\section{Materials and methods}

\section{Samples}

In total, 154 nontumoral and 550 tumoral samples of human tissues were collected from the archives of the Pathology Department from the 'Nuestra Señora del Prado' (Talavera de la Reina-Toledo), 'Complejo Hospitalario Universitario de Vigo' (CHUVI)' (VigoPontevedra) and 'Virgen de la Salud' (Toledo) Hospitals, and from the tissue archives of the CNIO Tumour Bank Network. Paraffin blocks were selected on the basis of the availability of suitable formalin-fixed paraffin-embedded tissue (at least 1-mm thick). This study was carried out on anonymized archival biopsy material. The procedure complied with the guidelines of the Helsinki Declaration and the study was approved by the Ethics Committee of the Hospital Carlos III (National Center for Clinical Research).

Three different samples of each of the following nontumoral human samples were collected:

- Central nervous system: parietal lobes, cerebellum, basal nuclei, brain stem, choroid plexus.

- Gastrointestinal tract: esophagus, stomach, small bowel, colon, appendix, liver, pancreas, gallbladder.

- Respiratory tract: parotid, larynx, trachea, lung (pleura and bronchi).

- Endocrine system: pituitary, thyroid, parathyroid and adrenal glands.

- Skin and soft tissue: skin, muscle.

- Lymphoid tissue: tonsil, spleen, thymus.

- Breast and gynecological tissue: breast, ovary, uterus (cervix, endometrium, myometrium), fallopian tubes, placenta, umbilical cord.

- Male reproductive system and urinary tract: kidney, bladder, prostate, testis, seminal vesicles, epididymis.

- Hyperplasic and inflammatory tissue: nodular thyroid hyperplasia, parathyroid hyperplasia, 
seborrheic keratosis, ulcerative colitis, hyperplasic polyp of the colon.

The tumoral samples included in the study represent the most frequent tumor types in humans, and include the following diagnoses:

- Central nervous system: low- and high-grade astrocytomas, oligodendroglioma, ependymoma, meningioma (10 cases per type).

- Gastrointestinal tract and annexes: gastric adenocarcinoma (10 well differentiated and eight poorly differentiated cases); gastric signet-ring adenocarcinoma (10 cases), colonic villous adenoma (10 low-grade and 10 high-grade dysplasia cases); colonic tubular adenoma (10 cases); well-differentiated colorectal adenocarcinoma (10 cases); mucinous colorectal adenocarcinoma (10 cases), poorly differentiated colorectal adenocarcinoma (5 cases); hepatocellular carcinoma (10 cases); gallbladder adenocarcinoma (9 cases); carcinoid tumor of the appendix (3 cases); endocrine pancreatic tumor (5 cases); exocrine pancreatic adenocarcinoma (5 cases).

- Respiratory tract tumors: parotid pleomorphic adenoma (10 cases), squamous cell carcinoma of the larynx (10 cases), lung adenocarcinoma (10 cases), lung squamous cell carcinoma (10 cases), lung undifferentiated large-cell carcinoma (10 cases), lung neuroendocrine small-cell carcinoma (6 cases), mesothelioma (10 cases).

- Endocrine tumors: pituitary adenoma (10 cases), thyroid papillary carcinoma (10 cases), thyroid follicular carcinoma (8 cases), thyroid medullary carcinoma (9 cases), adrenal cortex carcinoma (3 cases), adrenal pheochromocytoma (8 cases), parathyroid adenoma (10 cases).

- Skin and soft tissue tumors: cutaneous basal cell carcinoma (10 cases), cutaneous squamous cell carcinoma (10 cases), malignant melanoma (10 cases), capillary haemangioma (10 cases), Kaposi's sarcoma (10 cases), fibrous solitary tumor (4 cases), leiomyosarcoma (6 cases), liposarcoma (7 cases) and atypical lipomas (3 cases).

- Lymphomas: non-Hodgkin's lymphomas (follicular, mantle cell (MCL), diffuse large B-cell. Burkitt's (BL) and peripheral T-cell lymphomas) and Hodgkin's lymphomas (HL) (10 cases of each type).

- Breast and gynecological tumors: breast ductal carcinoma (10 cases), breast lobular carcinoma (8 cases), squamous cell carcinoma of the cervix (10 cases), endometrial adenocarcinoma (8 cases) and serous endometrial carcinoma ( 2 cases), uterus leiomyoma (4 cases); ovary mucinous cystadenoma (5 cases) and cystadenocarcinomas (4 cases), ovary serous cystadenomas (4 cases) and cystadenocarcinomas (6 cases), ovary endometrioid adenocarcinoma (7 cases) and cystadenocarcinomas (3 cases), ovary clear-cell carcinoma (10 cases).

- Tumors of the urinary and male reproductive system: bladder urothelial carcinoma, clear-cell renal-cell carcinoma (10 cases), prostate adenocarcinoma (10 cases), seminoma (10 cases), embryonal carcinoma (5 cases), teratocarcinoma (5 cases).

\section{Tissue-Microarray Design}

We used a Tissue Arrayer device (Beecher Instrument, Silver Spring, MD, USA) to construct the tissue microarrays (TMAs). All case samples were histologically reviewed and the most representative areas for each tissue or tumor type were marked in the paraffin blocks. Two selected 1-mm-diameter cylinders from two different areas were included in the TMAs. Thus, 10 different TMA blocks were constructed, two with normal tissue and eight with tumoral samples, each containing between 120 and 180 cylinders. Several controls were included in each block (placenta, testis, tonsil and tumoral cell lines) as internal controls to ensure the quality, reproducibility and homogenous staining of the slides.

The percentage of informative individual cores depends on the antibody evaluated. As each TMA included two different core cylinders for each case, the final percentage of missing expression data values was $10 \%$ for RING1, $5 \%$ for RNF2, $16 \%$ for BMI1, $11 \%$ for MEL18, $6 \%$ for RYBP and $3 \%$ for HPH1.

\section{Immunohistochemical Techniques}

The procedure has been described elsewhere. ${ }^{39,42}$ Immunohistochemical staining was performed on these sections using the following antibodies (Abs): ${ }^{42}$ HPH1- $\alpha$-mouse-monoclonal (Mc) Ab, RNF2 (3 $\alpha$ )-mouse-McAb, RING1-rabbit-polyclonal (Pc) Ab, RYBP-rabbit-PcAb, BMI1 goat-PcAb (C20) (Santa Cruz Biotechnology Inc., CA, USA), MEL18 goatPcAb (C20) (Santa Cruz) and for Ki67 the MIB1 antibody (DAKO, Glostrup, Denmark). Conditions for the demonstration of these proteins and controls for the antibodies have been described in other reports. ${ }^{7,8,29,48,42}$ Staining of TMA sections was evaluated by three different pathologists (ES, JG-C and $\mathrm{MM}$ ), using uniform criteria. Discrepancies in the scoring of cases were resolved after joint examination on a multi-headed microscope. In order to guarantee the reproducibility of this method, we decided to employ straightforward, clearcut criteria, and cases were scored as positive ( 1 or 2 ) or negative (0) for every antibody (Table 1 ).

\section{Extraction of Nuclei from Paraffin-Embedded Tissues}

To study RING1 loss by FISH, we separated nuclei from paraffin cores, according to the protocol described by Paternoster et al. ${ }^{49}$ Briefly, we marked representative tumor areas on H\&E-stained sections. Corresponding areas were identified on the paraffin 
Table 1 Proteins and antibodies used in the study

\begin{tabular}{lllllll}
\hline Protein & Clone & Source & Detection & Reactivity & Threshold (1/2) & Internal control \\
\hline RING1 $^{7,42}$ & RABBIT-Pc & MV & LSAB/DAB & $0,1,2$ & $25 / 50 \%$ cells & Reactive lymphocytes, endothelial cells \\
RNF2 $^{42,48}$ & Mc (3- $\alpha)$ & HK & LSAB/DAB & $0,1,2$ & $25 / 50 \%$ cells & Reactive lymphocytes, endothelial cells \\
BMI1 $^{42}$ & GOAT-Pc (C20) & SC & ENVIS/DAB & $0,1,2$ & $25 / 50 \%$ cells & Reactive lymphocytes, endothelial cells \\
MEL18 $^{29,42}$ & GOAT-Pc (C20) & SC & LSAB/DAB & $0,1,2$ & $25 / 50 \%$ cells & Reactive lymphocytes, plasma cells \\
RYBP $^{8,42}$ & RABBIT-Pc & MV & LSAB/DAB & $0,1,2$ & $10 / 25 \%$ cells & TMA controls (placenta) \\
HPH1 $^{22,56}$ & Mc $(\alpha)$ & HK & LSAB/DAB & $0,1,2$ & $10 / 25 \%$ cells & TMA controls (germinal cells in testis) \\
KI67 & MIB1-Mc & DAKO & ENVIS/DAB & $0,1,2$ & $25 / 50 \%$ cells & Proliferating cells \\
\hline
\end{tabular}

Source, detection, reactivity, threshold and controls are indicated.

SC, Santa Cruz Biotechnology Inc. (CA, USA); HK, produced by H Koseki; MV, produced by M Vidal; Pc, polyclonal; Mc, monoclonal; DAB, diaminobenzidine; LSAB, LSAB Visualization System (DAKO, Glostrup, Denmark); TMA, tissue microarray.

For HPH1 and RYBP, 1: 10-25\% positive cells; 2: more than $25 \%$ positive cells.

For all others, 1: $25-50 \%$ positive cells; 2 : more than $50 \%$ positive cells.

blocks, and core tissue biopsies of 0.6-mm diameter were taken from each block using a tissue microarrayer needle (Beecher Instrument). The paraffin was dissolved at room temperature with three 20-min changes of xylene. The tissue was then rehydrated with 100, 95, 75 and $50 \%$ ethanol (EtOH) for $15 \mathrm{~min}$ each. The $50 \% \mathrm{EtOH}$ was removed and the tissue was manually disaggregated with the tip of a paper clip. The tissue cores were digested by adding $100 \mu \mathrm{l}$ of freshly prepared proteinase $\mathrm{K}$ solution (DAKO) and incubated at $37^{\circ} \mathrm{C}$ for $1-2 \mathrm{~h}$ depending on the tissue type. To aid enzymatic digestion, the sample was vortexed for $3 \mathrm{~s}$ several times during this incubation period. Nuclei were pelleted using a mini-microcentrifuge (7000 r.p.m.) for $10 \mathrm{~min}$. Proteinase $\mathrm{K}$ was carefully removed with a micropipette and the nuclei washed in phosphatebuffered saline (PBS). The PBS solution was removed and the nuclei fixed by resuspension with vortexing of freshly prepared fixative (three parts methanol and one part glacial acetic acid). The nuclei were resuspended in $50 \mu \mathrm{l}$ of fixative. Fixed nuclei suspensions were stored at $-70^{\circ} \mathrm{C}$ until it was convenient to perform FISH studies.

Cytospins on glass slides were prepared by centrifugation of $25-\mu \mathrm{l}$ cell suspensions $+100 \mu \mathrm{l}$ glacial acetic acid $70 \%$ (10 min, 1000 r.p.m.) in a Shandon Cytospin3 cytocentrifuge (Thermo Electron Corporation). The cytospin preparations were air-dried for 2-24h. The samples were immediately processed for FISH, or stored in sealed boxes at $-20^{\circ} \mathrm{C}$ before performing FISH.

\section{Fluorescence In Situ Hybridization (FISH)}

FISH analysis was carried out for the detection of BMI1 and RING1 copy number changes as previously reported for HER2 and $c-M Y C C^{50}$ In short, we used 4-mm sections of the TMAs for BMI1 and the cytospin described above for RING1 analysis. For the study of BMI1 amplification, we used the Bacterial Artificial Chromosome (BAC) clone RP11-573G6, from Research Genetics, Invitrogen Corp. (Carlsbad, CA, USA), which spans the entire 10p12.2 genomic region, together with a commercial centromeric probe for chromosome 10 (Vysis, Inc., DownersGrove, IL; USA) that was used as a control for the ploidy level for chromosome 10. For the study of RING1 losses, we used the BAC clone RP11731H07, from Research Genetics, Invitrogen Corp., which spans the entire $6 \mathrm{p} 21.3$ genomic region, together with a commercial centromeric probe for chromosome 6 (Vysis, Inc).

Slides were deparaffinized, boiled in a pressure cooker with $1 \mathrm{mM}$ EDTA, pH 8.0 for $10 \mathrm{~min}$ and incubated with pepsin at $37^{\circ} \mathrm{C}$ for $30 \mathrm{~min}$. The slides were then dehydrated. Probes were denatured at $75^{\circ} \mathrm{C}$ for 2 min after overnight hybridization at $37^{\circ} \mathrm{C}$ in a humid chamber. Slides were washed with $0.4 \times$ SSC and $0.3 \%$ NP40.

FISH evaluation was performed by two investigators (MCM and JCC) with no previous knowledge of other genetic, clinical or immunohistochemical results. Fluorescence signals were scored in each sample by counting the number of single-copy gene and centromeric signals in an average of 130 (60$210)$ well-defined nuclei. Amplification was defined as the presence (in $>5 \%$ of tumor cells) of either $>10$ gene signals or more than three times as many gene signals as centromere signals of the chromosome. Deletion was defined as the absence of one signal of the gene compared with the centromeric probe signal from the same chromosome. Cutoff values for the copy-number changes were obtained from the analysis of normal adjacent cells in each experiment.

\section{Results}

\section{PcG Protein Expression in Normal Tissues}

The first objective was to investigate the distribution of PcG proteins in normal human tissues to establish the expression pattern of PcG in nontumoral tissues. To this end, we studied the expression of several PcG members of the PcGm complex (RING1, RNF2, BMI1, MEL18, HPH1 and RYBP) in 154 samples of 
Table 2 Summary of PcG expression in normal tissues (For more information see Supplementary Table SI.1)

RING1 RNF2 BMI1 MEL18 RYBP $H P H 1$

\begin{tabular}{|c|c|c|c|c|c|c|}
\hline Testis & ++ & + & + & + & - & + \\
\hline Placenta & ++ & + & + & + & ++ & - \\
\hline Thyroid gland & ++ & + & + & + & + & - \\
\hline SNC & ++ & + & + & + & - & - \\
\hline Pancreas & ++ & + & + & ++ & - & + \\
\hline Skin & ++ & + & + & + & - & - \\
\hline Tonsil & ++ & + & + & + & - & - \\
\hline Spleen & ++ & + & + & + & & \\
\hline Thymus & ++ & + & + & + & - & - \\
\hline Breast & ++ & + & + & + & - & - \\
\hline Kidney & ++ & + & + & + & - & - \\
\hline Pituitary gland & ++ & - & - & + & + & + \\
\hline Parathyroid & ++ & - & ++ & + & - & + \\
\hline Adrenal gland & ++ & - & + & + & - & - \\
\hline Stomach & ++ & - & + & + & - & - \\
\hline Small intestine & ++ & - & + & + & - & - \\
\hline Colon & ++ & - & + & + & - & - \\
\hline Parotid gland & ++ & - & + & + & - & - \\
\hline Prostate & ++ & - & + & + & - & - \\
\hline Uterus & ++ & - & + & + & - & - \\
\hline Respiratory tract & ++ & - & - & + & - & - \\
\hline Liver & ++ & - & - & + & - & - \\
\hline Ovary & ++ & + & + & - & - & - \\
\hline Striated muscle & ++ & - & - & - & - & - \\
\hline
\end{tabular}

nontumoral adult human tissues grouped in two TMAs.

The results revealed great variability in the distribution of most of the proteins in the tissues, and a very selective expression of some of these PcG components. Some tissues, such as testis, placenta and thyroid gland, expressed most of the proteins, while others, such as lung, liver and striated muscle, seemed to express only one or two proteins (Table 2 and Supplementary Table SI.1).

The intensity and cellular-type distribution were different for each protein. In placenta, for example, the cytotrophoblast cells expressed MEL18, RNF2, BMI1 and RING1 and syncytiotrophoblast cells expressed RING1 and RYBP. HPH1 is the only PcG protein that was not detected in placenta (Table 2 and Supplementary Table SI.1).

Concerning the selective distribution of PcG proteins, some of them, such as RING1, were widely expressed in almost every tissue and cell type, while others were detected exclusively in a few specific cell types. For instance, RYBP was found in placenta, umbilical cord, thyroid gland and scattered cells in the pituitary gland, and HPH1 could be found in Langerhans islets, pituitary and parathyroid gland and germ cells in the testis. The results are summarized in Table 2 and Supplementary Table SI.1, and some examples are illustrated in Figure 1a, c, e and g.

Due to the proposed role of PcG in the control of cellular proliferation, Ki67, a marker of cellular proliferation, was also analyzed in these tissues. Levels of this marker were not clearly correlated with the presence of any PcG protein in tumors. Nevertheless, in normal lymphoid tissue, BMI1 was detected in quiescent cells (mantle cells) and MEL18 was present in the proliferating cells of the germinal center (GC) (Supplementary Table SI.1).

\section{PcG Protein Expression in Human Tumors}

The main goal of this study was to identify abnormalities in the expression of these proteins in human cancer, and, consequently, the role PcG protein deregulation may play in human tumorigenesis. Thus, we analyzed the expression of PcG proteins in the most frequent human tumors by immunohistochemistry to evaluate the anomalies in the expression of this system in tumors compared with normal tissues. We collected 550 tumoral samples (see 'Samples' in Material and Methods section) and made eight TMAs. A synopsis of the results is presented in Figure 2 and Supplementary Table SI.2.

Changes in the expression of PcG between tumors and their normal-cell counterparts

Overall, tumor samples maintained the expression pattern of their normal counterparts. In agreement with this, RING1 protein was the most ubiquitously detected PcG protein in human tumors, and RYBP and HPH1 were undetectable in most tumors. However, some degree of heterogeneity was found in the staining for all proteins in specific tumors. The most significant observations made in this series are described below:

RING1: As previously mentioned, this protein was detected in every normal tissue type. When the tumors were analyzed, this ubiquitous expression was preserved with few notable exceptions: it was absent from only $3 \%$ of the tumors, the negative cases being of clear-cell renal-cell carcinoma (4/8 cases) (Figure 1h) and testicular germ-cell tumors (9/ 18), the latter including teratocarcinomas (1/4), embryonal carcinomas (2/4) (Figure 1d), and seminomas (6/10). These data are even more interesting given that RING1 was detected in the normal counterpart of the tumors, both in tubule cells in the kidney (Figure 1g) and in germ cells in the testis (Figure 1C).

RNF2: Analysis of the multitumor TMAs revealed some discrepancies between tumoral tissues and their normal counterparts. Such was the case for gastrointestinal tumors, whereby $77 \%$ of gastric $(23 / 28)$ and $70 \%(40 / 55)$ of colonic tumors expressed RNF2, while it was not detected in the stomach or colon surface-epithelial cells (Figure 1e and f). GC-derived lymphomas (diffuse large B-cell lymphoma (DLBCL), Burkitt's lymphoma (BL) and Hodgkin's lymphomas (HL)) also had a higher level of RNF2 expression than that detected in B-GC cells. 

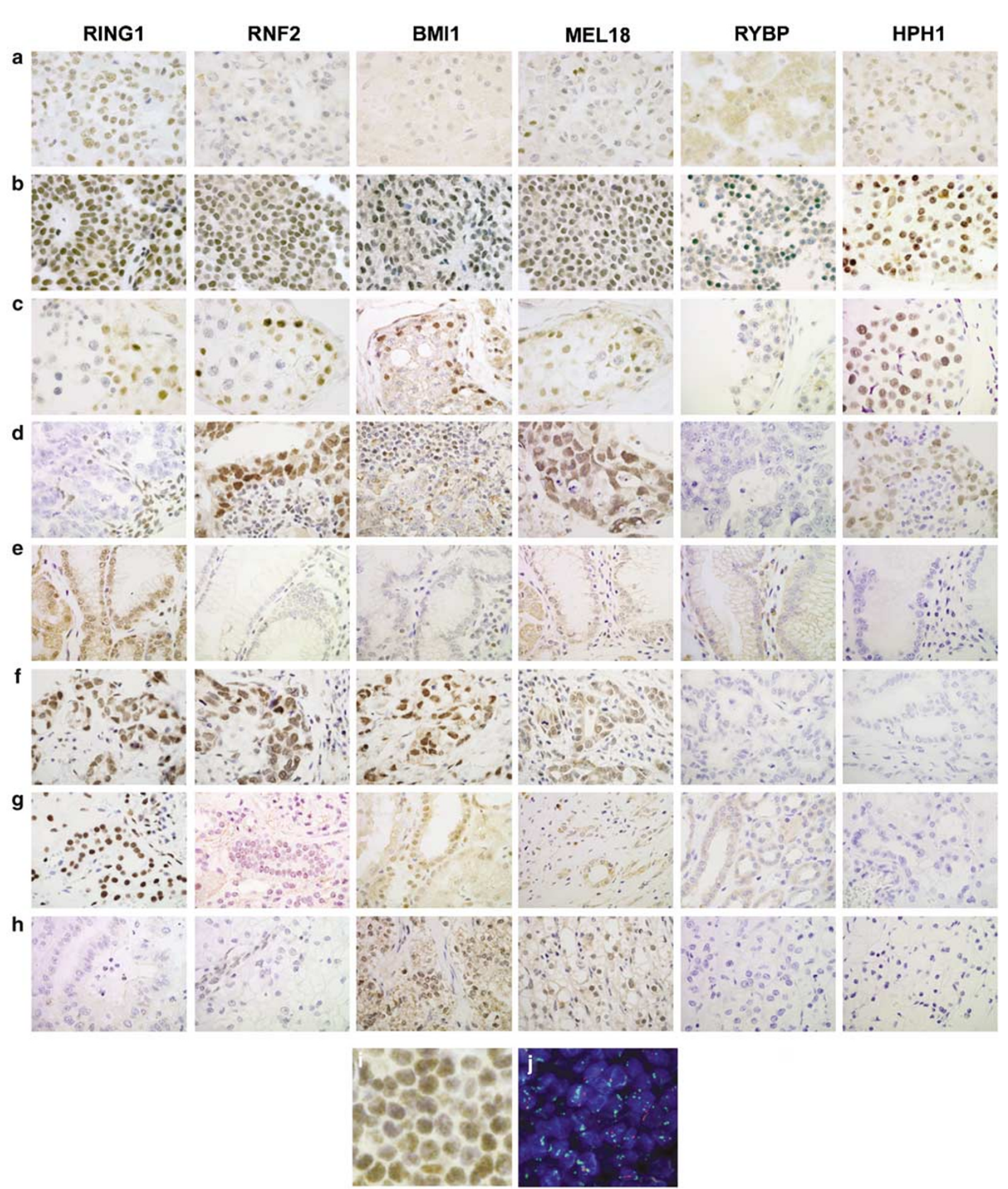

Figure 1 Examples of different patterns of PcG expression in normal and tumoral tissues: (a) pituitary gland; (b) pituitary adenoma; (c) testis; (d) embryonal carcinoma; (e) gastric surface-epithelial cells; (f) gastric adenocarcinoma; (g) kidney tubules; (h) clear-cell renal-cell carcinoma. (i and j) BMI1 amplification detected by FISH in an MCL (j) case overexpressing BMI1 (i). The picture shows a group of cells with several copies of BMI1 (stained in green) in comparison with two centromeric copies for chromosome 10 (red).

BMI1: The expression of BMI1 was also variable in normal tissues and tumors, whereby $77 \%$ of tumors were positive for this protein. In gastrointestinal tumors, its expression compared with that of normal tissues was similar to that of RNF2. Accordingly, $63 \%$ of gastrointestinal tumors expressed BMI1, 


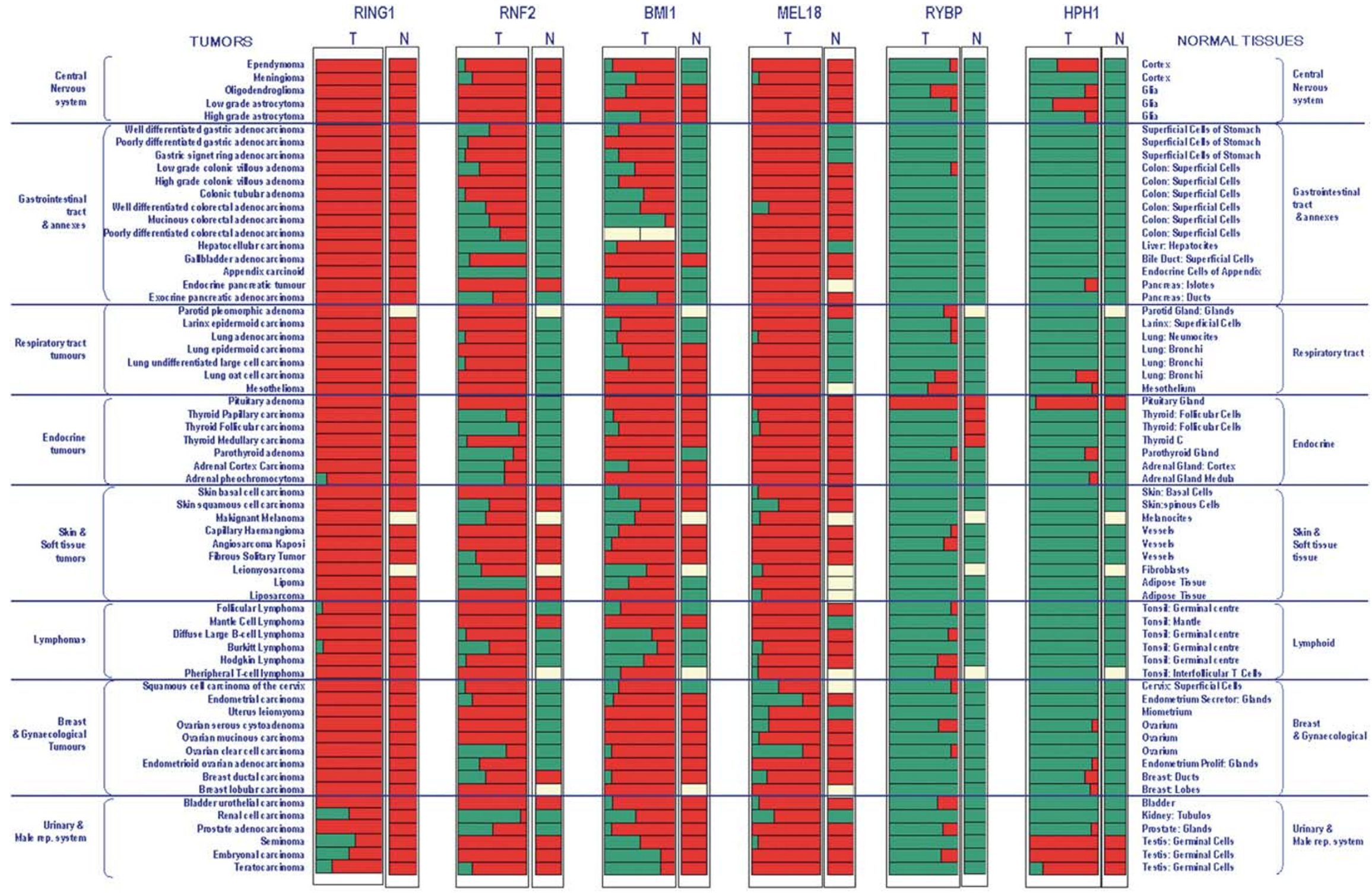

Figure 2 Expression of PcG proteins in human tumors (T) and in the corresponding normal tissue counterparts (N). Red color represents positive expression; green indicates the absence of expression. In the case of the tumors, the length of the red/green bars is proportional to the proportion of positive/negative cases, respectively, for each tumor type. Yellow indicates no samples. 
whereas it was only detected in sporadic colonic or gastric surface-epithelial cells (Figure 1e and f). BMI1 was also detected in several GC-derived lymphomas (DLBCL, BL and HL), whereas it was not expressed in nontumoral GC B-cells (Figure 2). MCL, pituitary adenomas and parathyroid adenomas were the tumors that expressed the highest levels of BMI1 protein compared to those detected in their normal cell counterparts (Supplementary Table SI.2).

BMI1 was not detected in 6/18 testicular germ-cell tumors (Figure 1d), and 4/9 renal-cell carcinomas (Figure 1h), whereas it was detected in germ cells in the testis (Figure 1c) and in tubule cells in the kidney (Figure 1g).

MEL18: This protein, together with RING1, was the most ubiquitously expressed in human tumors. Only $8 \%$ of the tumors were found to be negative for this protein. The tumoral types with a higher percentage of negative samples were cutaneous squamous-cell carcinoma (4/10), ovary clear-cell carcinoma (6/8), endometrial adenocarcinoma (6/8) and squamous-cell carcinoma of the cervix (4/10). MEL18 was detected in the corresponding normal counterparts (Figure 2).

$R Y B P$ : Only $10 \%$ of all tumor cases were found to be positive for RYBP, the cases corresponding to the following: oligodendroglioma (4/10), pituitary adenomas (9/9), Hodgkin (3/10) and T-cell lymphomas (3/9). RYBP was not detected in glial cells, or in normal lymphoid B- or T-cells.

HPH1: $10 \%$ of the tumoral cases were positive for HPH1 protein. In the central nervous system, HPH1 was detected in 6/9 low-grade compared with $2 / 10$ high-grade astrocytoma cases, and in oligodendroglioma $(2 / 10)$ and ependymoma (6/10). The normal glial cells did not express the protein. HPH1 was also detected in 9/10 pituitary adenomas, and in nearly every testicular germ-cell tumor (18/19) (Figure 1d). HPH1 protein was also detected in the pituitary gland and the germ cells of the testis.

\section{FISH study}

The level of BMI1 protein expression was found to be particularly high in three tumor types: MCL (6/10), pituitary adenoma (9/9) and parathyroid adenomas (8/10). Such a level of expression has previously been associated with genomic amplification in MCL. ${ }^{36,37}$ Therefore, we decided to study this possibility in these three tumors types by FISH analysis in TMA paraffin sections. Our findings confirmed amplification of BMI1 locus in MCL in two of 10 cases, six of them with high BMI1 protein expression, and in one case out of nine pituitary adenomas. No amplification was detected in any of the 10 parathyroid adenomas analyzed, eight of which had high BMI1 levels.

As mentioned before, the loss of RING1 expression, only observed in $3 \%$ of all the tumors analyzed, was very selective for germinal-cell tumors and clear-cell renal-cell carcinomas. There- fore, we also employed FISH analysis to look for loss of RING1 locus in these tumors. To do this, we decided to separate nuclei from the whole paraffin sections of the tumors included in the TMAs as described in the Material and Methods section. In total, 16 cases were analyzed in this way: 13 negative cases and three positives as controls (clear-cell renal-cell carcinoma (five negative/two positive), seminomas (6/0), teratocarcinomas (1/1) and embryonal carcinomas (2/0)). Loss of RING1 locus was not detected in any case.

\section{Unique PcG protein expression in pituitary} adenomas, clear-cell renal-cell carcinomas and germ-cell tumors

There are some examples of tumors with a unique PcG expression pattern (Figures 1 and 2).

Testicular germ-cell tumors showed a distinctive PcG pattern with HPH1 expression at the highest levels and a parallel absence of RING1 protein and also of BMI1 (Figure 1d).

Renal-cell carcinoma was the other tumor type lacking RING1 expression, and also frequently showing absence of RNF2, BMI1 and MEL18. HPH1 was not detected in these tumors (Figure 1h).

Pituitary adenoma was the only type of tumor that coexpressed every PcG protein analyzed in this study (Figure 1b). Every valid case expressed all the proteins, except one that was negative for HPH1 (Supplementary Table SI.2).

\section{Discussion}

Our analysis of PcG protein distribution in human normal and tumoral tissue allows us to identify a PcG protein-expression profile in human normal tissues and tumors that implies a role for some PcG genes in specific neoplasms. Additionally, the anomalous high level of expression of BMI1 associated with gene amplification strongly supports a role for this PcG protein in cancer.

The distribution of several PcG proteins differed greatly in normal human tissues and among different cell types in specific tissues. HPH1 was detected exclusively in germ cells in testis, in pituitary and parathyroid gland and the pancreatic islets of Langerhans, and RYBP was found in placenta, umbilical cord, thyroid and pituitary glands. By contrast, RING1 was ubiquitously expressed in normal tissues, although some variability among different tissues and cell types was observed. MEL18, BMI1 and RNF2 had a more variable tissue distribution.

Overall, tumor samples shared the same expression pattern as their normal counterpart, although changes in the expression of PcG proteins associated with tumoral transformation were found for each protein at least in some tumors. There were several particularly remarkable findings associated with this tumoral transformation: (a) RING1-loss, which 




Figure 3 Proposed PcG components of the PcGm complex in several normal and tumoral tissues. The expression of various PcG proteins in different cell types allows us to propose the presence of different components of PcGm members in distinct normal and tumoral tissues.

seemed to be a very selective finding in clear-cell renal-cell carcinoma and testicular germ-cell tumors: (b) MEL18 loss, which was characteristically found in several gynecological tumors, such as squamous-cell carcinoma of the cervix, uterine endometrial adenocarcinoma and ovarian clear-cell adenocarcinoma. This finding is consistent with the previously described loss of MEL18 in tumoral cell lines. $^{51}$ (c) In contrast, other PcG proteins were overexpressed in a number of tumors. This was the case for RNF2 and BMI1, which were both detected in gastrointestinal tumors or GC-derived lymphomas, according to previously published data. ${ }^{37,44}$ Although BMI1 was detected in mantle cells in reactive tonsils, its levels in MCL were also anomalously high, consistent with the amplification of this gene ${ }^{36,37}$ confirmed in this study by FISH. High levels of BMI1 were also detected for the first time in pituitary and parathyroid adenomas, and gene amplification was confirmed in pituitary adenomas; and (d) HPH1 and RYBP were detected, with a mutually exclusive pattern, in tumors of the central nervous system. RYBP was also found in HL, as previously described, ${ }^{42}$ and in peripheral T-cell lymphomas.

The remarkable unique PcG expression profiles observed in particular tumors merit special attention. Testicular germ-cell tumors were one such type, with an absence of RING1 protein expression in $50 \%$ of cases, and a loss of BMI 1 in $67 \%$ of cases. These are the tumors that expressed HPH1 most frequently, in consonance with the observations made in normal germ cells, expressing the highest levels of HPH1. Clear-cell renal-cell carcinoma is another tumor type lacking RING1 expression that frequently features an absence of RNF2, BMI1 and MEL18.

Pituitary adenomas had a very singular and distinctive PcG expression profile since they were the only tumor type expressing the six PcG proteins analyzed in almost every case. Additionally, strong expression of BMI1 was detected in all these cases, and was associated with gene amplification in one of them.

One of the points to be explored arising from these observations is that the composition of the PcG complex could vary among different cell types and could be regulated during tumoral transformation. This has been proposed in earlier studies ${ }^{16,52}$ and our findings support the existence of tissue and cellspecific PcG complexes. Even the proteins grouped in the same PcG complex, as in the case of the proteins included in the present study, which belonged to the PcGm complex, could form different complexes depending on the cell type or differentiation status, and during tumor development (Figure 3).

The selective distribution of the different components of the PcG complex in normal tissues and tumors could help to clarify their role as epigenetic chromatin modifiers, since changes in the methylation status, including those of nucleosomal histones, have been proposed as being universal in cancer cells. ${ }^{53}$

These data are evidence of the significant involvement that has been proposed for BMI1, by which it plays a role in permitting the self-renewal capacity of cancer stem cells. ${ }^{23,43,54}$ The relatively generalized increase in the expression of BMI1 in different tumor types compared with their normal counterpart cell types and the gene amplification detected in some tumors in the present study, sustains the interpretation that BMI1 occupies a key position in cancer development. These results provide no evidence in support of the proposed hypothetical role $^{55}$ for BMI1 or MEL18 as cell-cycle regulators in tumors.

\section{Acknowledgements}

We are indebted to María J Acuña and Raquel Pajares for their expertise and excellent technical assistance with TMA technology and immunohistochemical assays. We also thank Juan L Orradre, José 
Palacios, Francisca I Camacho, and Soledad Alonso for the evaluation of the samples, and Laura Cereceda from the Tumour Bank Network at the CNIO for her help with the collection of samples for the TMAs. This work was supported by grants from the Ministerio de Ciencia y Tecnología, Spain (SAF2001-2211; SAF2001-0060; SAF2004-06952C02-02).

\section{References}

1 Pirrotta V. Polycomb silencing and the maintenance of stable chromatin states. Results Probl Cell Differ 1999; 25:205-228.

2 Mahmoudi T, Verrijzer CP. Chromatin silencing and activation by Polycomb and trithorax group proteins. Oncogene 2001;20:3055-3066.

3 Jacobs JJ, van Lohuizen M. Polycomb repression: from cellular memory to cellular proliferation and cancer. Biochim Biophys Acta 2002;1602:151-161.

4 Satijn DP, Otte AP. RING1 interacts with multiple Polycomb-group proteins and displays tumorigenic activity. Mol Cell Biol 1999;19:57-68.

5 Hashimoto N, Brock HW, Nomura M, et al. RAE28, BMI1, and M33 are members of heterogeneous multimeric mammalian Polycomb group complexes. Biochem Biophys Res Commun 1998;245:356-365.

6 Alkema MJ, Bronk M, Verhoeven E, et al. Identification of Bmi1-interacting proteins as constituents of a multimeric mammalian polycomb complex. Genes Dev 1997;11:226-240.

7 Schoorlemmer J, Marcos-Gutierrez C, Were F, et al. Ring1A is a transcriptional repressor that interacts with the Polycomb-M33 protein and is expressed at rhombomere boundaries in the mouse hindbrain. EMBO J 1997;16:5930-5942.

8 Garcia E, Marcos-Gutierrez C, Lorente MdM, et al. RYBP, a new repressor protein that interacts with components of the mammalian Polycomb complex, and with the transcription factor YY1. EMBO J 1999;18:3404-3418.

9 Wang H, Wang L, Erdjument-Bromage H, et al. Role of histone $\mathrm{H} 2 \mathrm{~A}$ ubiquitination in Polycomb silencing. Nature 2004;431:873-878.

10 Sewalt RG, van der Vlag J, Gunster MJ, et al. Characterization of interactions between the mammalian polycomb-group proteins Enx1/EZH2 and EED suggests the existence of different mammalian polycomb-group protein complexes. Mol Cell Biol 1998;18: 3586-3595.

11 Czermin B, Melfi R, McCabe D, et al. Drosophila enhancer of Zeste/ESC complexes have a histone H3 methyltransferase activity that marks chromosomal Polycomb sites. Cell 2002;111:185-196.

12 Breiling A, Turner BM, Bianchi ME, et al. General transcription factors bind promoters repressed by Polycomb group proteins. Nature 2001;412:651-655.

13 Trimarchi JM, Fairchild B, Wen J, et al. The E2F6 transcription factor is a component of the mammalian Bmi1-containing polycomb complex. Proc Natl Acad Sci USA 2001;98:1519-1524.

14 van der Vlag J, Otte AP. Transcriptional repression mediated by the human polycomb-group protein EED involves histone deacetylation. Nat Genet 1999;23: 474-478.
15 van Lohuizen M. Functional analysis of mouse Polycomb group genes. Cell Mol Life Sci 1998;54:71-79.

16 Lessard J, Baban S, Sauvageau G. Stage-specific expression of polycomb group genes in human bone marrow cells. Blood 1998;91:1216-1224.

17 Akasaka T, Tsuji K, Kawahira $\mathrm{H}$, et al. The role of mel-18, a mammalian Polycomb group gene, during IL-7-dependent proliferation of lymphocyte precursors. Immunity 1997;7:135-146.

18 Lessard J, Schumacher A, Thorsteinsdottir U, et al. Functional antagonism of the Polycomb-Group genes eed and Bmi1 in hemopoietic cell proliferation. Genes Dev 1999;13:2691-2703.

19 van der Lugt NM, Domen J, Linders K, et al. Posterior transformation, neurological abnormalities, and severe hematopoietic defects in mice with a targeted deletion of the bmi-1 proto-oncogene. Genes Dev 1994;8:757-769.

20 Voncken JW, Roelen BA, Roefs M, et al. Rnf2 (Ring1b) deficiency causes gastrulation arrest and cell cycle inhibition. Proc Natl Acad Sci USA 2003;100: 2468-2473.

21 Raaphorst FM, van Kemenade FJ, Fieret E, et al. Cutting edge: polycomb gene expression patterns reflect distinct $\mathrm{B}$ cell differentiation stages in human germinal centers. J Immunol 2000;164:1-4.

22 Raaphorst FM, Otte AP, van Kemenade FJ, et al. Distinct BMI-1 and EZH2 expression patterns in thymocytes and mature $\mathrm{T}$ cells suggest a role for Polycomb genes in human $\mathrm{T}$ cell differentiation. J Immunol 2001;166:5925-5934.

23 Park IK, Qian D, Kiel M, et al. Bmi-1 is required for maintenance of adult self-renewing haematopoietic stem cells. Nature 2003;423:302-305.

24 Kajiume T, Ninomiya Y, Ishihara H, et al. Polycomb group gene mel-18 modulates the self-renewal activity and cell cycle status of hematopoietic stem cells. Exp Hematol 2004;32:571-578.

25 Molofsky AV, Pardal R, Iwashita $\mathrm{T}$, et al. Bmi-1 dependence distinguishes neural stem cell selfrenewal from progenitor proliferation. Nature 2003; 425:962-967.

26 Lessard J, Sauvageau G. Bmi-1 determines the proliferative capacity of normal and leukaemic stem cells. Nature 2003;423:255-260.

27 Simon JA, Tamkun JW. Programming off and on states in chromatin: mechanisms of Polycomb and trithorax group complexes. Curr Opin Genet Dev 2002;12: 210-218.

28 Visser HP, Gunster MJ, Kluin-Nelemans HC, et al. The Polycomb group protein EZH2 is upregulated in proliferating, cultured human mantle cell lymphoma. Br J Haematol 2001;112:950-958.

29 van Galen JC, Dukers DF, Giroth C, et al. Distinct expression patterns of polycomb oncoproteins and their binding partners during the germinal center reaction. Eur J Immunol 2004;34:1870-1881.

30 Kleer CG, Cao Q, Varambally S, et al. EZH2 is a marker of aggressive breast cancer and promotes neoplastic transformation of breast epithelial cells. Proc Natl Acad Sci USA 2003;100:11606-11611.

31 Rhodes DR, Sanda MG, Otte AP, et al. Multiplex biomarker approach for determining risk of prostatespecific antigen-defined recurrence of prostate cancer. J Natl Cancer Inst 2003;95:661-668.

32 Varambally S, Dhanasekaran SM, Zhou M, et al. The polycomb group protein EZH2 is involved in progression of prostate cancer. Nature 2002;419:624-629. 
33 Haupt Y, Alexander WS, Barri G, et al. Novel zinc finger gene implicated as myc collaborator by retrovirally accelerated lymphomagenesis in $\mathrm{E}$ mu-myc transgenic mice. Cell 1991;65:753-763.

34 Haupt Y, Bath ML, Harris AW, et al. bmi-1 transgene induces lymphomas and collaborates with myc in tumorigenesis. Oncogene 1993;8:3161-3164.

35 Jacobs JJ, Scheijen B, Voncken JW, et al. Bmi-1 collaborates with c-Myc in tumorigenesis by inhibiting c-Myc-induced apoptosis via INK4a/ARF. Genes Dev 1999;13:2678-2690.

36 Kohlhammer $\mathrm{H}$, Schwaenen C, Wessendorf S, et al. Genomic DNA-chip hybridization in t(11;14)-positive mantle cell lymphomas shows a high frequency of aberrations and allows a refined characterization of consensus regions. Blood 2004;pp:795-801.

37 Bea S, Tort F, Pinyol M, et al. BMI-1 gene amplification and overexpression in hematological malignancies occur mainly in mantle cell lymphomas. Cancer Res 2001;61:2409-2412.

38 Dukers DF, van Galen JC, Giroth C, et al. Unique polycomb gene expression pattern in Hodgkin's lymphoma and Hodgkin's lymphoma-derived cell lines. Am J Pathol 2004;164:873-881.

39 Garcia JF, Camacho FI, Morente M, et al. Hodgkin and Reed-Sternberg cells harbor alterations in the major tumor suppressor pathways and cell-cycle checkpoints: analyses using tissue microarrays. Blood 2003; 101:681-689.

40 Raaphorst FM, van Kemenade FJ, Blokzijl T, et al. Coexpression of BMI-1 and EZH2 polycomb group genes in Reed-Sternberg cells of Hodgkin's disease. Am J Pathol 2000;157:709-715.

41 Schwering I, Brauninger A, Klein U, et al. Loss of the B-lineage-specific gene expression program in Hodgkin and Reed-Sternberg cells of Hodgkin lymphoma. Blood 2003;101:1505-1512.

42 Sanchez-Beato M, Sanchez E, Garcia JF, et al. Abnormal PcG protein expression in Hodgkin's lymphoma. Relation with E2F6 and NFkappaB transcription factors. J Pathol 2004;204:528-537.

43 Leung C, Lingbeek M, Shakhova O, et al. Bmi1 is essential for cerebellar development and is overexpressed in human medulloblastomas. Nature 2004; 428:337-341.

$44 \mathrm{Kim} \mathrm{JH}$, Yoon SY, Kim CN, et al. The Bmi-1 oncoprotein is overexpressed in human colorectal cancer and correlates with the reduced p16INK4a/ p14ARF proteins. Cancer Lett 2004;203:217-224.
45 Vonlanthen S, Heighway J, Altermatt HJ, et al. The bmi-1 oncoprotein is differentially expressed in nonsmall cell lung cancer and correlates with INK4A-ARF locus expression. Br J Cancer 2001;84:1372-1376.

46 van Kemenade FJ, Raaphorst FM, Blokzijl T, et al. Coexpression of BMI-1 and EZH2 polycomb-group proteins is associated with cycling cells and degree of malignancy in B-cell non-Hodgkin lymphoma. Blood 2001;97:3896-3901.

47 Raaphorst FM, Vermeer M, Fieret E, et al. Site-specific expression of polycomb-group genes encoding the HPC-HPH/PRC1 complex in clinically defined primary nodal and cutaneous large B-cell lymphomas. Am J Pathol 2004;164:533-542.

48 Suzuki M, Mizutani-Koseki Y, Fujimura Y, et al. Involvement of the Polycomb-group gene Ring1B in the specification of the anterior-posterior axis in mice. Development 2002;129:4171-4183.

49 Paternoster SF, Brockman SR, McClure RF, et al. A new method to extract nuclei from paraffin-embedded tissue to study lymphomas using interphase fluorescence in situ hybridization. Am J Pathol 2002;160: 1967-1972.

50 Palacios J, Honrado E, Osorio A, et al. Immunohistochemical characteristics defined by tissue microarray of hereditary breast cancer not attributable to BRCA1 or BRCA2 mutations: differences from breast carcinomas arising in BRCA1 and BRCA2 mutation carriers. Clin Cancer Res 2003;9:3606-3614.

51 Matsuo F, Yano K, Saito H, et al. Mutation analysis of the mel-18 gene that shows decreased expression in human breast cancer cell lines. Breast Cancer 2002;9: 33-38.

52 Gunster MJ, Raaphorst FM, Hamer KM, et al. Differential expression of human Polycomb group proteins in various tissues and cell types. J Cell Biochem 2001;81:129-143.

53 Lund AH, van Lohuizen M. Epigenetics and cancer. Genes Dev 2004;18:2315-2335.

54 Valk-Lingbeek ME, Bruggeman SW, van Lohuizen M. Stem cells and cancer; the polycomb connection. Cell 2004;118:409-418.

55 Jacobs JJ, Kieboom K, Marino S, et al. The oncogene and Polycomb-group gene bmi-1 regulates cell proliferation and senescence through the ink4a locus. Nature 1999;397:164-168.

56 Atsuta T, Fujimura S, Moriya $\mathrm{H}$, et al. Production of monoclonal antibodies against mammalian Ring1B proteins. Hybridoma 2001;20:43-46.

Supplementary information accompanies the paper on Modern Pathology website (http://www.nature.com/ modpathol) 